\title{
The endogenous cannabinoid system in the gut of patients with inflammatory bowel disease
}

\author{
A Di Sabatino ${ }^{1,7}, \mathrm{~N}_{\text {Battista }}{ }^{2,7}, \mathrm{P}_{\text {Biancheri }}{ }^{3}, \mathrm{C} \mathrm{Rapino}^{2}$, L Rovedatti $^{1}, \mathrm{G} \mathrm{Astarita}^{4}$, A Vanoli $^{1}$, E Dainese $^{2}$, \\ M Guerci $^{1}$, D Piomelli ${ }^{4}$, SLF Pender ${ }^{5}$, TT MacDonald ${ }^{3}$, M Maccarrone M $^{2,6,8}$ and GR Corazza $^{1,8}$
}

Activation of cannabinoid receptors (CBs) by endocannabinoids impacts on a number of gastrointestinal functions. Recent data indicate that CB1 agonists improve 2,4-dinitrobenzene sulfonic acid-induced colitis in mice, thus suggesting a role for the endocannabinoid agonist anandamide (AEA) in protecting the gut against inflammation. We here examined the gut endocannabinoid system in inflammatory bowel disease (IBD) patients, and investigated the ex vivo and in vitro effects of the non-hydrolysable AEA analog methanandamide (MAEA) on the mucosal proinflammatory response. The content of AEA, but not of 2-arachidonoyl-glycerol and $N$-palmitoylethanolamine, was significantly lower in inflamed than uninflamed IBD mucosa, and this was paralleled by lower activity of the AEA-synthesizing enzyme $\mathrm{N}$-acyl-phosphatidylethanolamine-specific phospholipase $\mathrm{D}$ and higher activity of the AEA-degrading enzyme fatty acid amide hydrolase. MAEA significantly downregulated interferon $-\gamma$ and tumor necrosis factor- $\alpha$ secretion by both organ culture biopsies and lamina propria mononuclear cells. Although these results are promising, further studies are needed to determine the role of cannabinoid pathways in gut inflammation.

\section{INTRODUCTION}

Inflammatory bowel diseases (IBD), which encompass Crohn's disease and ulcerative colitis, are driven by an excessive immune response in the gut. ${ }^{1,2}$ Despite recent therapeutic advances and improved knowledge of the underlying pathogenic mechanisms, patients with IBD are often resistant to treatment, justifying the continued search for new therapeutic approaches. ${ }^{3}$ Recently, understanding of the nature and regulation of endogenous cannabinoids in the gut has suggested that the endocannabinoid system might be a therapeutic target in IBD. ${ }^{4-6}$

The cannabinoid system comprises specific G-protein-coupled receptors (CB1 and $\mathrm{CB} 2$ ), a variety of exogenous (marijuanaderived cannabinoids) and endogenous ligands, and a machinery dedicated to endocannabinoid synthesis and degradation. ${ }^{7}$ Endocannabinoids include amides and esters of long-chain polyunsaturated fatty acids, among which anandamide (AEA) and 2-arachidonoylglycerol (2-AG) are the main endogenous agonists of $\mathrm{CB} 1$ and $\mathrm{CB} 2 .{ }^{8} \mathrm{CB} 1$ are predominant in brain and vessels and mediate the psychoactive effects of cannabis, whereas CB2 are normally expressed on immune cells. ${ }^{9}, 10$ AEA shows higher affinity for $\mathrm{CB} 1$ over $\mathrm{CB} 2$, whereas 2-AG binds equally well to both CB1 and CB2. ${ }^{7-9}$ Other endocannabinoid-like compounds have been described to date, such as $\mathrm{N}$-palmitoylethanolamine (PEA) and oleoylethanolamide, which are unable to bind with significant affinity to either CB1 and CB2. ${ }^{11}$

Endocannabinoid signaling is terminated by specific degradation systems involving their cellular uptake by a facilitated transport mechanism and their hydrolysis by fatty acid amide hydrolase (FAAH) for AEA, monoacylglycerol lipase for 2-AG, and $N$-acylethanolamine-hydrolyzing acid amidase for PEA.,11 Although AEA synthesis might be because of several metabolic routes, $N$-acyl-phosphatidylethanolamine-specific phospholipase $\mathrm{D}$ (NAPE-PLD) is currently considered the major enzyme responsible for AEA production, ${ }^{12}$ whereas 2 -AG synthesis is mainly mediated by diacylglycerol lipase. ${ }^{7,13}$ Several synthetic compounds have been shown to efficiently block either endocannabinoid uptake or degradation, thus providing

\footnotetext{
${ }^{1}$ First Department of Medicine, Fondazione IRCCS Policlinico S. Matteo, Centro per lo Studio e la Cura delle Malattie Infiammatorie Croniche Intestinali, University of Pavia, Pavia, Italy. ${ }^{2}$ Department of Biomedical Sciences, University of Teramo, Teramo, Italy. ${ }^{3}$ Centre for Immunology and Infectious Disease, Blizard Institute of Cell and Molecular Science, Barts and the London School of Medicine and Dentistry, London, UK. ${ }^{2}$ Department of Pharmacology, University of California, Irvine, California, USA. 5Division of Infection, Inflammation and Repair, University of Southampton, Southampton, UK. ${ }^{6}$ European Center for Brain Research (CERC)/Santa Lucia Foundation, Rome, Italy. ${ }^{7}$ Joint first authors. ${ }^{8}$ Joint last authors. Correspondence: A Di Sabatino or M Maccarrone (a.disabatino@smatteo.pv.it or mmaccarrone@unite.it) 
pharmacological tools to enhance the activity of the endocannabinoid system. ${ }^{14}$

Besides their effects on central nervous system and on gastrointestinal motility, the anti-inflammatory properties of Cannabis sativa preparations have long been known, and potential roles of endocannabinoids in modulating the immune response have been recently reviewed. ${ }^{15-17}$ In particular, AEA downregulates interleukin (IL)-2 transcription and secretion, reduces tumor necrosis factor (TNF)- $\alpha$ and interferon (IFN) $-\gamma$ production, induces lymphocyte apoptosis, and inhibits neutrophil recruitment at the site of inflammation. ${ }^{17}$ Inflammatory stimuli may in turn control endocannabinoids by regulating their degradation. Indeed, lipopolysaccharide stimulation increases lymphocyte intracellular content of AEA by downregulating FAAH gene expression. ${ }^{18}$

There is evidence from experimental studies that the endocannabinoid system is involved in intestinal inflammation. $4,5,15,19$ In particular, FAAH-deficient mice are protected from 2,4-dinitrobenzene sulfonic acid-induced colitis, whereas a pharmacological blockade of CB1 leads to a worsening of murine colitis. ${ }^{20}$ On this basis, we first examined the levels of endocannabinoids and their receptors in IBD inflamed and uninflamed mucosa, and then investigated the ex vivo and in vitro effects of the synthetic non-hydrolysable AEA analog methanandamide (MAEA) on proinflammatory cytokine production by gut biopsies and lamina propria mononuclear cells (LPMCs) from IBD patients. Furthermore, we explored the action of MAEA on myofibroblasts isolated from Crohn's disease strictures.

\section{RESULTS}

\section{In vivo mucosal endocannabinoid levels}

Mucosal levels of AEA, 2-AG, and PEA were assessed by highperformance liquid chromatography (HPLC)-mass spectrometry (Figure 1a). Median levels of AEA were significantly lower in IBD inflamed mucosa (in Crohn's disease: $46 \mathrm{pmol} \mathrm{g}^{-1}$, range 15-89; in ulcerative colitis: $71 \mathrm{pmol} \mathrm{g}^{-1}$, range 36-286, $P<0.05$ ) in comparison with IBD uninflamed mucosa (in Crohn's disease: $123 \mathrm{pmol} \mathrm{g}^{-1}$, range 61-427; in ulcerative colitis: $122 \mathrm{pmol} \mathrm{g}^{-1}$, range 63-675). No significant difference was found in AEA levels between Crohn's disease and ulcerative colitis uninflamed mucosa and normal colon $\left(131 \mathrm{pmol} \mathrm{g}^{-1}\right.$, range 21-542). Unlike AEA, 2-AG median levels were comparable in IBD inflamed mucosa (in Crohn's disease: $5.0 \mathrm{nmolg}^{-1}$, range 1.2-8.5; in ulcerative colitis: $6.5 \mathrm{nmol} \mathrm{g}^{-1}$, range 2.1-19.5), IBD uninflamed mucosa (in Crohn's disease: $4.6 \mathrm{nmol} \mathrm{g}^{-1}$, range 1.0-8.1; in ulcerative colitis: $5.2 \mathrm{nmol} \mathrm{g}^{-1}$, range $2.1-11.5$ ) and normal colon $\left(4.0 \mathrm{nmol} \mathrm{g}^{-1}\right.$, range 1.5-9.1). PEA median concentration did not significantly differ in IBD inflamed mucosa (in Crohn's disease: $123 \mathrm{pmol} \mathrm{g}^{-1}$, range $57-1,127$; in ulcerative colitis: 151 pmol g $^{-1}$, range 55-380), IBD uninflamed mucosa (in Crohn's disease: $163 \mathrm{pmol} \mathrm{g}^{-1}$, range 54-677; in ulcerative colitis: $310 \mathrm{pmol} \mathrm{g}^{-1}$, range 55-1,394), and normal colon $\left(109 \mathrm{pmol} \mathrm{g}^{-1}\right.$, range $\left.28-401\right)$. Based on these results, we further investigated the activity of AEA-metabolic enzymes. Among the 34 control subjects, no difference was found in the mucosal levels of AEA, 2-AG, and PEA between those who turned out to have functional diarrhea at the end of their diagnostic work-up and those undergoing colectomy for colon cancer.

\section{In vivo mucosal enzyme activity}

The activity of NAPE-PLD and FAAH, which respectively synthesize and degrade AEA, was assessed by radiochromatography (Figure 1b). Median NAPE-PLD activity was significantly lower in IBD inflamed mucosa (in Crohn's

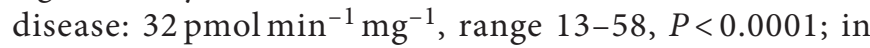
ulcerative colitis: $63 \mathrm{pmol} \mathrm{min}^{-1} \mathrm{mg}^{-1}$, range $17-116, P<0.01$ ) in comparison with IBD uninflamed mucosa (in Crohn's disease: $138 \mathrm{pmol} \mathrm{min}^{-1} \mathrm{mg}^{-1}$, range $89-150$; in ulcerative colitis: 120 pmol min ${ }^{-1} \mathrm{mg}^{-1}$, range $83-186$ ). No significant difference was found in NAPE-PLD activity between IBD uninflamed mucosa and normal colon $\left(193 \mathrm{pmol} \mathrm{min}^{-1} \mathrm{mg}^{-1}\right.$, range 74-208). On the contrary, median FAAH activity was significantly higher in IBD inflamed mucosa (in Crohn's disease: $820 \mathrm{pmol} \mathrm{min}^{-1} \mathrm{mg}^{-1}$, range $361-886, P<0.001$; in ulcerative colitis: $711 \mathrm{pmol} \mathrm{min}^{-1} \mathrm{mg}^{-1}$, range $\left.275-881, P<0.005\right)$ in comparison with IBD uninflamed mucosa (in Crohn's disease: $184 \mathrm{pmol} \mathrm{min}^{-1} \mathrm{mg}^{-1}$, range 73-680; in ulcerative colitis: $241 \mathrm{pmol} \mathrm{min}^{-1} \mathrm{mg}^{-1}$, range 80-651). No significant difference was found in FAAH activity between IBD uninflamed mucosa and normal colon (median $206 \mathrm{pmol} \mathrm{min}^{-1} \mathrm{mg}^{-1}$, range 98911). Among the 34 control subjects, no difference was found in NAPE-PLD and FAAH activity between those who turned out to have functional diarrhea at the end of their diagnostic work-up and those undergoing colectomy for colon cancer.

\section{In vivo mucosal $\mathrm{CB} 1$ and $\mathrm{CB} 2$ expression}

$\mathrm{CB} 1$ and $\mathrm{CB} 2$ expression was investigated through enzymelinked immunosorbent assay (ELISA; Figure 2a), immunoblotting (Figure 2b), and immunohistochemistry (Figure 2c-f). CB1 concentration, detected by ELISA and expressed as a mean percentage of control subjects, was significantly higher in the inflamed mucosa of 14 IBD patients (in Crohn's disease: mean $262 \pm 10 \%, P<0.001$; in ulcerative colitis: mean $204 \pm 13 \%$, $P<0.05$ ) in comparison with the uninflamed mucosa (in Crohn's disease: mean $100 \pm 14 \%$; in ulcerative colitis: mean $100 \pm 14 \%$; Figure 2a). These results were confirmed both by immunoblotting experiments, which showed a higher expression of CB1 in inflamed than uninflamed mucosa of both Crohn's disease and ulcerative colitis patients (Figure 2b), and immunohistochemistry, which showed increased CB1 expression by both epithelial cells and LPMCs in inflamed IBD mucosa (Figure 2d) in comparison with IBD uninflamed mucosa (Figure 2c). On the contrary, no significant difference was found in $\mathrm{CB} 2$ levels detected by ELISA between inflamed IBD mucosa (in Crohn's disease: mean 106 $\pm 10 \%$; in ulcerative colitis: mean $205 \pm 12 \%$ ) and uninflamed IBD mucosa (in Crohn's disease: mean $100 \pm 19 \%$; in ulcerative colitis: mean $100 \pm 5 \%$; Figure 2b). Accordingly, no difference was found in CB2 expression detected both by immunoblotting in inflamed mucosa in comparison with uninflamed mucosa, both in ulcerative colitis and Crohn's disease patients (Figure 2b), and immunohistochemistry that showed a similar pattern of CB2 expression in IBD uninflamed (Figure 2e) 


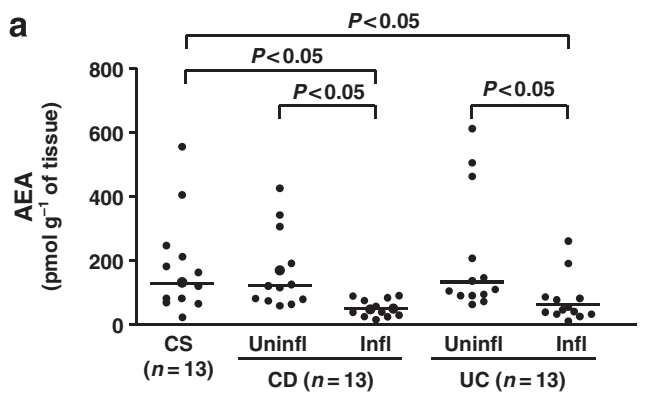

b
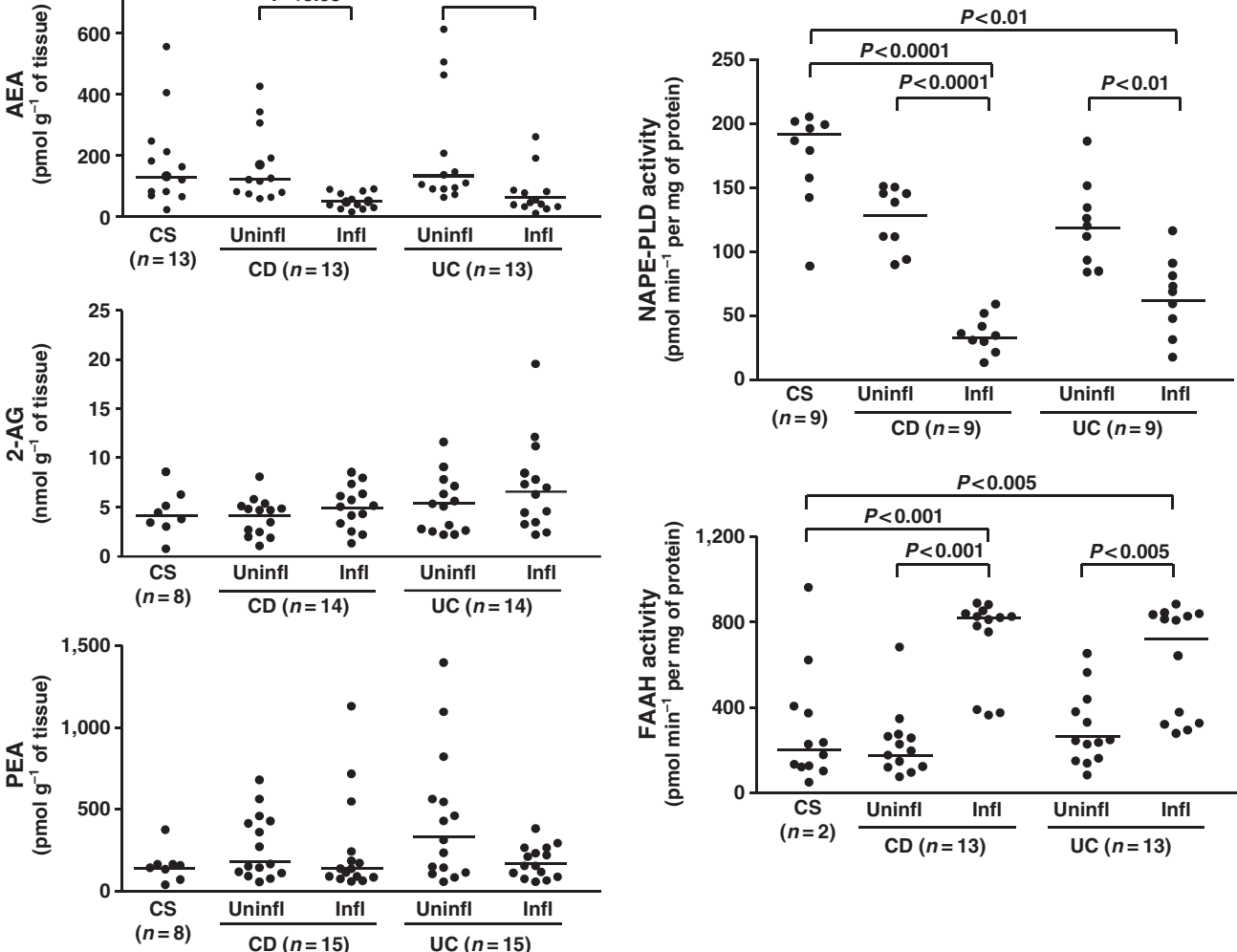

Figure 1 In vivo mucosal levels of endocannabinoids and anandamide (AEA)-synthesizing and AEA-degrading enzymes. (a) Levels of AEA, 2-arachidonoylglycerol (2-AG), and $\mathrm{N}$-palmitoylethanolamine (PEA) were measured by high-performance liquid chromatography (HPLC)-tandem mass spectrometry, and (b) activity of $\mathrm{N}$-acyl-phosphatidylethanolamine-specific phospholipase D (NAPE-PLD) and fatty acid amide hydrolase (FAAH) were assessed by radiochromatography, in the inflamed (Infl) and uninflamed (Uninfl) intestinal mucosa of Crohn's disease (CD) and ulcerative colitis (UC) patients, and in the normal gut of control subjects (CS).

and inflamed mucosa (Figure 2f), mostly limited to scattered mononuclear cells in the lamina propria. Among the 34 control subjects, no difference was found in CB1 and CB2 expression between those who turned out to have functional diarrhea at the end of their diagnostic work-up and those undergoing colectomy for colon cancer.

\section{In vitro and ex vivo effects of MAEA on proinflammatory cytokines}

To investigate the effect of the non-hydrolysable AEA analog MAEA on proinflammatory cytokine production, we measured IFN- $\gamma$ and TNF- $\alpha$ concentration in the supernatants of antiCD3/CD28-stimulated LPMCs from inflamed areas of 33 IBD patients ( 18 with Crohn's disease and 15 with ulcerative colitis) after incubation with increasing concentrations of MAEA (Figure 3a). As shown in Table 1a, both IFN- $\gamma$ and TNF- $\alpha$ production by stimulated IBD LPMCs were significantly inhibited in a concentration-dependent manner by MAEA. It is noteworthy that the maximal effect of MAEA was comparable with that exerted by FK506. No appreciable difference in the efficacy of MAEA between Crohn's disease and ulcerative colitis samples was observed. In addition, we investigated the effect of MAEA on IFN- $\gamma$ and IL-17 production by purified $\mathrm{CD}^{+}{ }^{+}$LPMCs from 16 patients with IBD ( 8 with Crohn's disease and 8 with ulcerative colitis), and we found that MAEA exerted a concentration-dependent downregulatory effect on IFN- $\gamma$ and IL-17 production by T cells (Table 1b). We then explored the influence of MAEA on proinflammatory cytokine production by organ culture biopsies from inflamed mucosa of the same patients (Figure 3b). A significant dose-dependent decrease of both IFN- $\gamma$ and TNF- $\alpha$ concentration was observed in the supernatants of IBD inflamed biopsies incubated with increasing concentrations of MAEA, in comparison with biopsies incubated with medium only: IFN- $\gamma$ (mean $344 \pm 65 \mathrm{pg} \mathrm{ml}^{-1}$ with medium alone vs. $69 \pm 24 \mathrm{pg} \mathrm{ml}^{-1}$ at $1 \mu \mathrm{M}(P<0.001)$ and $27 \pm 9 \mathrm{pg} \mathrm{ml}^{-1}$ at $10 \mu \mathrm{M}(P<0.0001))$ and TNF- $\alpha\left(\right.$ mean $132 \pm 26 \mathrm{pg} \mathrm{ml}^{-1}$ with medium alone vs. $52 \pm 12 \mathrm{pg} \mathrm{m}^{-1}$ at $1 \mu \mathrm{M}(P<0.01)$ and $23 \pm 9 \mathrm{pg} \mathrm{ml}^{-1}$ at $\left.10 \mu \mathrm{m}(P<0.001)\right)$. No appreciable difference in the efficacy of MAEA between Crohn's disease and ulcerative colitis samples was observed.

\section{In vitro effect of MAEA on T-bet and pSTAT4 expression}

We then tested whether MAEA influenced the expression of the transcription factor T-bet and the phosphorylated form of signal transducer and activator of transcription 4 (pSTAT4). AntiCD3/CD28-stimulated LPMCs from inflamed areas of $21 \mathrm{IBD}$ patients (11 with Crohn's disease and 10 with ulcerative colitis) were cultured in the presence or absence of $10 \mu \mathrm{m}$ MAEA and, 

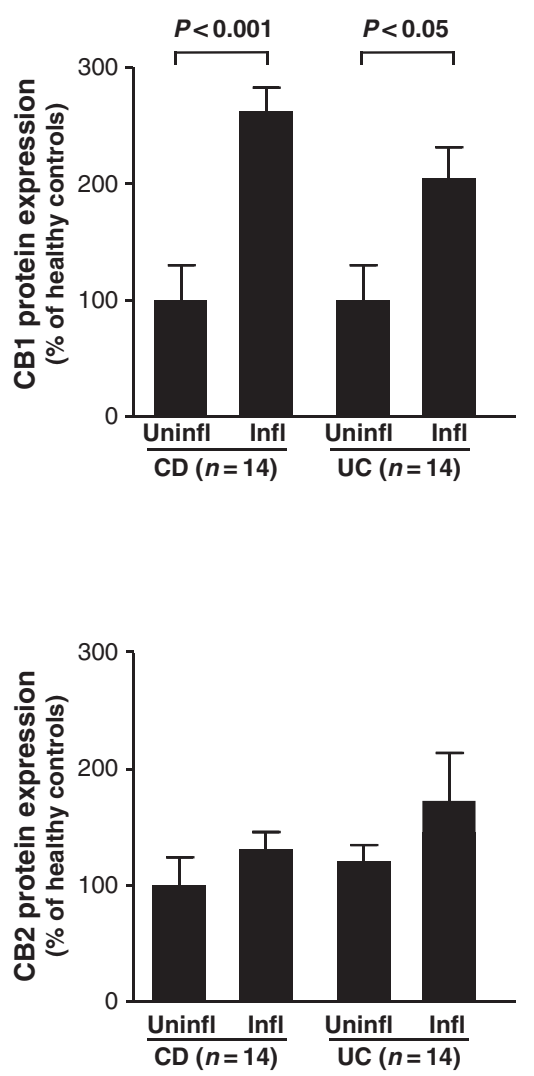

b
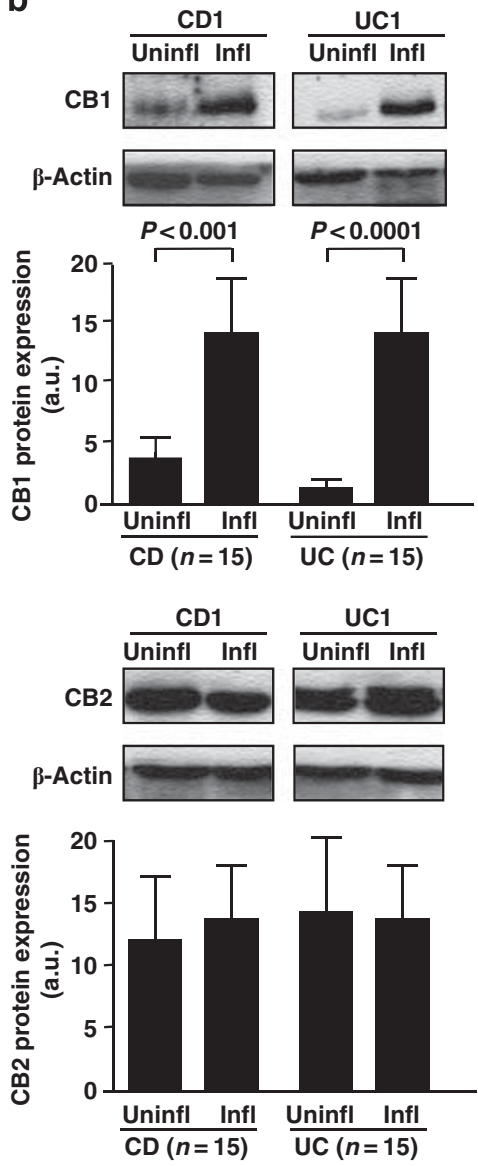
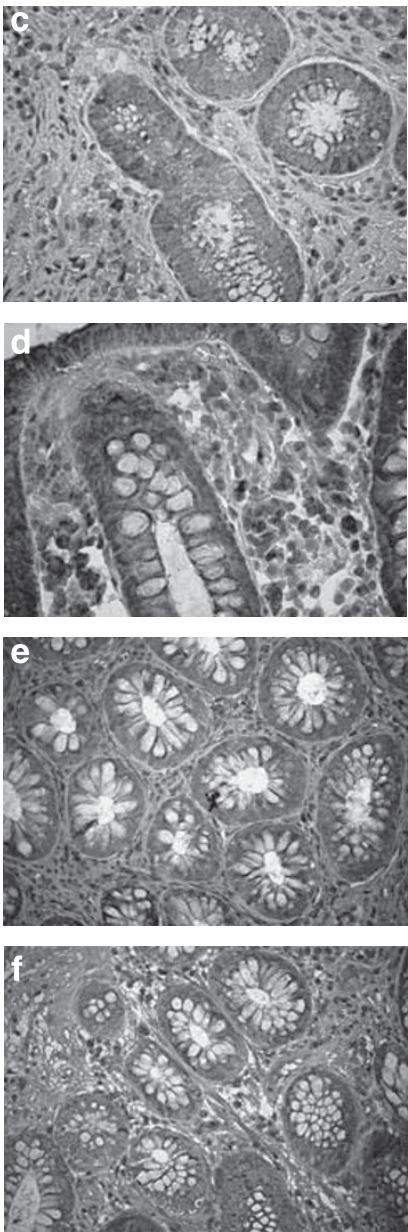

Figure 2 In vivo mucosal expression of type-1 (CB1) and type-2 (CB2) cannabinoid receptors. CB1 and CB2 expression was detected by (a) enzyme-linked immunosorbent assay (ELISA), (b) immunoblotting, and (c-f) immunohistochemistry in the inflamed (Infl) and uninflamed (Uninfl) mucosa of Crohn's disease (CD) and ulcerative colitis (UC) patients. (a) Results are expressed as mean percentage of control subjects \pm s.d. (b) Each example shown in the upper panel is representative of experiments performed in $15 \mathrm{CD}$ patients and $15 \mathrm{UC}$ patients. Blots were stripped and analyzed for $\beta$-actin as an internal loading control. The lower panel shows the densitometry of CB1 and CB2 expression normalized for $\beta$-actin. Results are mean \pm s.d. arbitrary units (a.u.). CB1 expression, which is evident at epithelial and lamina propria mononuclear cell level, is higher in (d) inflamed than in (c) uninflamed inflammatory bowel disease (IBD) mucosa. CB2 expression, which is limited to a few mononuclear cells in the lamina propria, is similar in (e) uninflamed and (f) inflamed IBD mucosa.

after culture, T-bet and pSTAT4 were determined by immunoblotting on cell lysates and respectively normalized for $\beta$-actin and total STAT4 (Figure 4a and $\mathbf{b})$. A significant $(P<0.001)$ increase of both T-bet and pSTAT4 expression was observed when LPMCs were stimulated with anti-CD3/CD28 antibodies. This increase was significantly $(P<0.001)$ inhibited by MAEA. No appreciable difference in the effect of MAEA on T-bet and pSTAT4 expression was observed between Crohn's disease and ulcerative colitis samples.

\section{In vitro effect of MAEA on Crohn's disease strictured myofibroblasts}

In order to assess the influence of the endocannabinoid system on intestinal fibrogenesis in Crohn's disease, we explored the effect of MAEA on collagen production and migration by myofibroblasts isolated from the submucosa of intestinal strictures of 11 patients with fibrostenosing Crohn's disease. Concentration of total soluble collagen was significantly $(P<0.01)$ lower (mean $140 \pm 33 \mu \mathrm{g} \mathrm{ml}^{-1}$ ) in the supernatant of myofibroblasts cultured with MAEA in comparison with those cultured with medium alone (mean $319 \pm 72 \mu \mathrm{g} \mathrm{ml}^{-1}$; Figure 5a). An in vitro wound-healing scratch assay was performed to assess the effects of MAEA on myofibroblast migration, which was measured as a percentage of wound repair. MAEA significantly $(P<0.05)$ increased the migration of Crohn's disease strictured myofibroblasts at $4 \mathrm{~h}$ (from mean $3.9 \pm 1.1$ to $10.9 \pm 1.4 \%$ ), $8 \mathrm{~h}$ (from mean $5.1 \pm 1.0$ to $15.1 \pm 1.6 \%$ ), $12 \mathrm{~h}$ (from mean $8.0 \pm 1.5$ to $20.3 \pm 3.5 \%$ ), $16 \mathrm{~h}$ (from mean $10.8 \pm 2.1$ to $21.9 \pm 1.3 \%$ ), $20 \mathrm{~h}$ (from mean $12.1 \pm 1.4$ to $24.1 \pm 3.5 \%$ ), and $24 \mathrm{~h}$ (from mean $15.2 \pm 1.5$ to $26.7 \pm 3.2 \%$ ) in comparison with myofibroblasts cultured with medium only evaluated at the same time points (Figure $5 \mathbf{b}$ ).

\section{DISCUSSION}

In this study we show that the content of the major endocannabinoid AEA is reduced in IBD inflamed mucosa as a consequence of both defective synthesis and increased degradation. The synthetic AEA analog MAEA exerts anti-inflammatory 
in vitro and ex vivo functions, as shown by the downregulation of proinflammatory cytokines following incubation of IBD LPMCs and biopsies with the synthetic compound. Moreover, MAEA inhibits collagen production and restores migration of myofibroblasts isolated from Crohn's disease strictures.

Endogenous cannabinoid agonists, together with the enzymes and receptors involved in their metabolism and signaling processes, are present in different tissues such as skin, brain, spleen, uterus, kidney, and gut, both in health and disease. ${ }^{8}$ There is increasing evidence that the endocannabinoid system plays an autoprotective role in immunologically mediated disorders. ${ }^{15,16}$ Nevertheless, most of the studies on this topic were conducted in animal models, and the way the endocannabinoid system exerts its protective activity in humans awaits further elucidation. We here detected AEA, 2-AG, and PEA levels in gut mucosa of IBD patients, and we found that AEA, but not 2-AG and PEA levels, are significantly reduced in inflamed compared with uninflamed areas, both in ulcerative colitis and Crohn's disease. We then asked the question whether this was secondary to a dysregulated AEA metabolism. On the basis of our results showing lower a

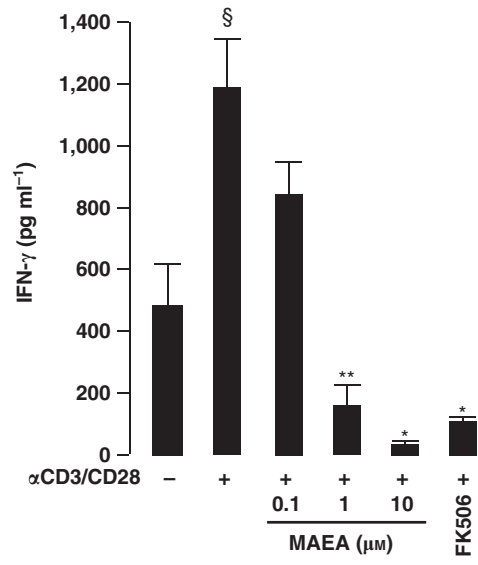

b

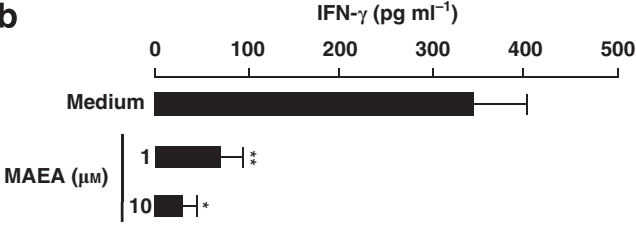

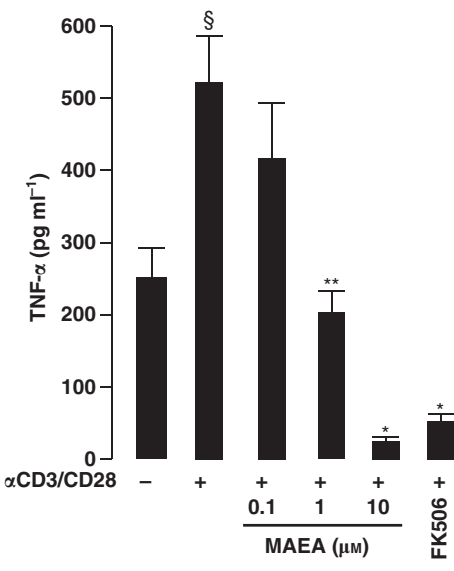

TNF- $\alpha\left(\mathrm{pg} \mathrm{ml}^{-1}\right)$

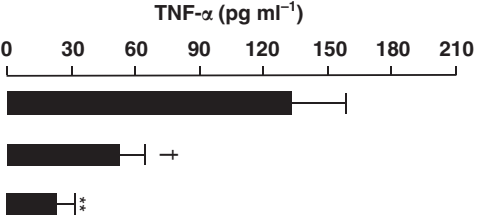

Figure 3 In vitro and ex vivo effects of methanandamide (MAEA) on proinflammatory cytokines. Interferon (IFN)- $\gamma$ and tumor necrosis factor (TNF)- $\alpha$ levels were detected by enzyme-linked immunosorbent assay (ELISA) (a) in the supernatants of lamina propria mononuclear cells (LPMCs) isolated from inflamed areas of 33 patients with inflammatory bowel disease (18 with Crohn's disease and 15 with ulcerative colitis), cultured for $48 \mathrm{~h}$ with antiCD3/CD28 antibodies $(\alpha C D 3 / C D 28)$ in the absence or presence of increasing concentrations of MAEA $(0.1-10 \mu \mathrm{M})$ or $10 \mu \mathrm{m}$ FK506, and (b) in the supernatants of biopsy specimens from inflamed areas of the same patients cultured for $24 \mathrm{~h}$ in the absence or presence of increasing concentrations of MAEA ( 1 and $10 \mu \mathrm{M}$ ). Results are mean \pm s.d. (a) ${ }^{\S} P<0.001$ vs. medium; ${ }^{\star} P<0.0001$ vs. $\alpha C D 3 / C D 28 ;{ }^{* \star} P<0.001$ vs. $\alpha C D 3 / C D 28 ;(b){ }^{\star} P<0.0001$ vs. medium only; ${ }^{\star \star} P<0.001$ vs. medium alone; ${ }^{\dagger} P<0.05$ vs. medium alone.

Table 1a Levels of IFN- $\gamma$ and TNF- $\alpha\left(\mathrm{pg} \mathrm{m}^{-1}\right)$ in the supernatants of anti-CD3/CD28-stimulated ( $\alpha$ CD3/CD28) LPMCs isolated from the inflamed colon of $18 \mathrm{CD}$ and $15 \mathrm{UC}$ patients, and cultured for $48 \mathrm{~h}$ with increasing concentrations $(0.1,1$, and $10 \mu \mathrm{M})$ of MAEA or with $10 \mu \mathrm{M}$ FK506

\begin{tabular}{|c|c|c|c|c|}
\hline Medium & $536(148)$ & $466(161)$ & 266 (33) & $238(51)$ \\
\hline$\alpha \mathrm{CD} 3 / \mathrm{CD} 28+10 \mu \mathrm{M}$ MAEA & $52(7)^{\star}$ & $34(12)^{*}$ & $35(7)^{\star}$ & $19(4)^{\star}$ \\
\hline$\alpha \mathrm{CD} 3 / \mathrm{CD} 28+1 \mu \mathrm{M}$ MAEA & $184(46)^{\star \star}$ & $156(32)^{\star \star}$ & $173(25)^{\star \star}$ & $229(36)^{\star \star}$ \\
\hline
\end{tabular}

Abbreviations: CD, Crohn's disease; IFN- $\gamma$, interferon- $\gamma$; LPMC, lamina propria mononuclear cell; MAEA, methanandamide; TNF- $\alpha$, tumor necrosis factor- $\alpha$; UC, ulcerative colitis.

Results are mean (s.d.)

$\S P<0.001$ vs. medium; ${ }^{\star} P<0.0001$ vs. $\alpha C D 3 / C D 28 ;{ }^{* \star} P<0.001$ vs. $\alpha C D 3 / C D 28$. 
Table $1 \mathrm{~b}$ Levels of IFN- $\gamma$ and IL-17 $\left(\mathrm{pg} \mathrm{ml}^{-1}\right)$ in the supernatants of anti-CD3/CD28-stimulated ( $\alpha$ CD3/CD28) T cells isolated from the inflamed colon of $8 \mathrm{CD}$ and $8 \mathrm{UC}$ patients, and cultured for $48 \mathrm{~h}$ with increasing concentrations $(0.1,1$, and $10 \mu \mathrm{M})$ of MAEA or with $10 \mu \mathrm{M}$ FK506

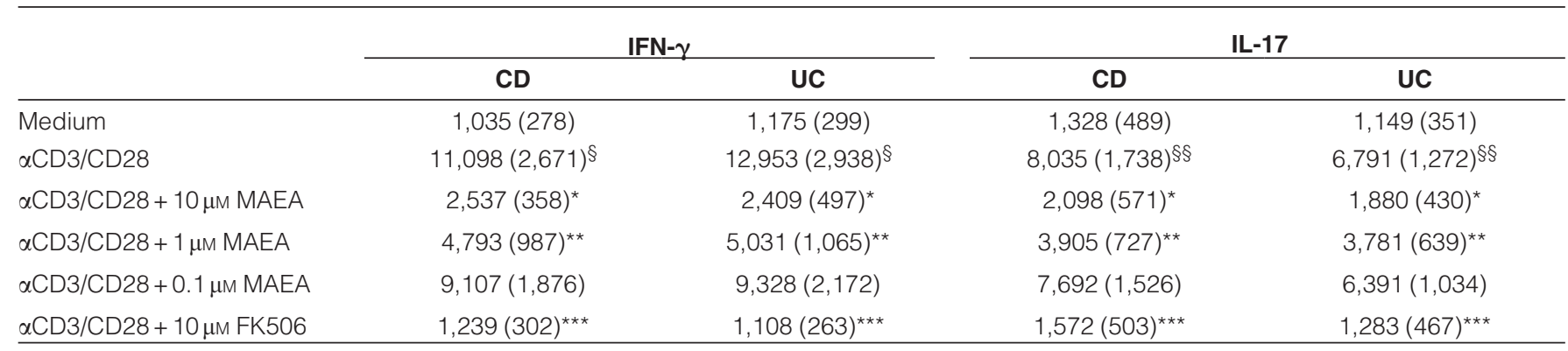

Abbreviations: CD, Crohn's disease; IFN- $\gamma$, interferon- $\gamma$; IL-17, interleukin-17; MAEA, methanandamide; UC, ulcerative colitis.

Results are mean (s.d.).

$\S P<0.0001$ vs. medium; $\$ \S P<0.001$ vs. medium; ${ }^{*} P<0.001$ vs. $\alpha C D 3 / C D 28 ;{ }^{* *} P<0.05$ vs. $\alpha C D 3 / C D 28 ;{ }^{* * *} P<0.0001$ vs. $\alpha C D 3 / C D 28$.
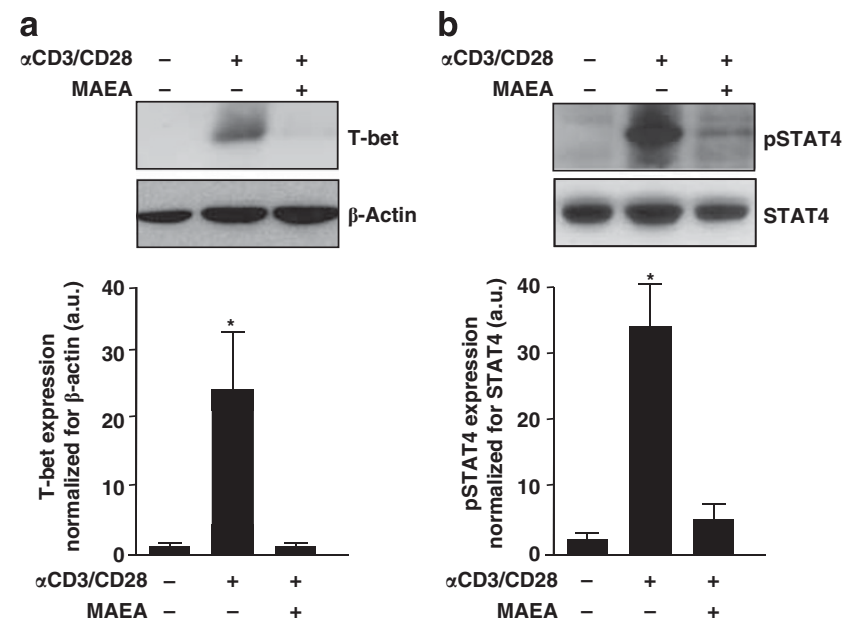

Figure 4 In vitro effect of methanandamide (MAEA) on the expression of T-bet and the phosphorylated form of signal transducer and activator of transcription 4 (pSTAT4) in lamina propria mononuclear cells isolated from inflamed areas of patients with inflammatory bowel disease.

(a) T-bet and (b) pSTAT4 were detected by immunoblotting of antiCD3/CD28 ( $\alpha \mathrm{CD} 3 / \mathrm{CD} 28$ )-stimulated lamina propria mononuclear cells incubated for $48 \mathrm{~h}$ in the absence or presence of $10 \mu \mathrm{m}$ MAEA. In the upper panels, each example is representative of experiments performed in 21 patients (11 with Crohn's disease and 10 with ulcerative colitis). Blots were stripped and analyzed for (a) $\beta$-actin or (b) total STAT4 as an internal loading control. The lower panels show (a) densitometry of T-bet normalized for $\beta$-actin, and (b) densitometry of pSTAT4 normalized for total STAT4. Results are mean \pm s.d. arbitrary units (a.u.). ${ }^{\star} P<0.001$.

NAPE-PLD activity and higher FAAH activity, we can conclude that reduced AEA levels result from both defective synthesis and increased degradation. FAAH can also degrade 2-AG in vitro, ${ }^{7,13}$ yet variations of its activity observed in IBD mucosa did not exert any effect on 2-AG levels. Our results are in agreement with the observation of Marquez et al., ${ }^{21}$ who found lower NAPE-PLD expression and high AEA levels in inflamed ulcerative colitis when compared with control mucosa, but they diverge from those by D'Argenio et al. ${ }^{22}$ Moreover, the findings of Marquez et al., ${ }^{21}$ who showed the lack of difference in the diacylglycerol lipase/monoacylglycerol lipase ratio between ulcerative colitis patient and healthy control epithelium, support our data on mucosal 2-AG levels.

We then assessed $\mathrm{CB}$ expression in mucosal homogenates, and we found a higher expression of CB1 but not CB2 in inflamed compared with uninflamed areas of both Crohn's disease and ulcerative colitis patients. CB1 stimulation reduces gastrointestinal inflammation in various animal models, ${ }^{23}$ and CB2 activation on immune cells downmodulates proinflammatory cytokine production. ${ }^{17,24-26} \mathrm{CB} 1$ is upregulated in the colon of mice treated with 2,4-dinitrobenzene sulfonic acid, ${ }^{20}$ and Börner et al. ${ }^{27}$ showed that anti-CD3/CD28-stimulation increases CB1, but not CB2, expression on human peripheral blood $\mathrm{T}$ cells. Numerous studies have shown a variable gut CB2 expression in animal models of colitis, either because they were performed in different species or because the protocols used diverged considerably. ${ }^{28}$ An overexpression of $\mathrm{CB} 2$ at the ulcerative margin in active Crohn's disease has been reported, ${ }^{28}$ which could be part of a redistribution of CB2 expression during inflammation, thus leaving the overall CB2 levels in the whole inflamed mucosa unchanged.

The endocannabinoid system plays a role in the modulation of several immune processes. ${ }^{15-17}$ Cannabinoids reduce the MHC class II expression on the surface of dendritic cells, hence affecting antigen presentation, and inhibit peripheral T-cell activation in response to lipopolysaccharide and antiCD3 antibodies. ${ }^{26,29,30}$ Based on these findings, several studies have focused on the use of cannabinoids for the treatment of immune-mediated diseases, both in experimental models and humans. ${ }^{29}$ As the dysregulated proinflammatory response observed in IBD pathogenesis is characterized by a $\mathrm{T}$ helper cell type (Th)1/Th17 immune profile, ${ }^{31}$ we explored the influence of endocannabinoids on T cell-mediated gut inflammation, by investigating the in vitro and ex vivo effects of the synthetic non-hydrolysable AEA analog MAEA on proinflammatory cytokine production by anti-CD3/CD28-stimulated LPMCs, $\mathrm{CD}^{+}$LPMCs, and biopsies from inflamed IBD mucosa. MAEA downregulated IFN- $\gamma$ and TNF- $\alpha$ production by LPMCs and biopsies in a concentration-dependent manner. Furthermore, MAEA exerted a direct immunomodulatory effect on T cells, 

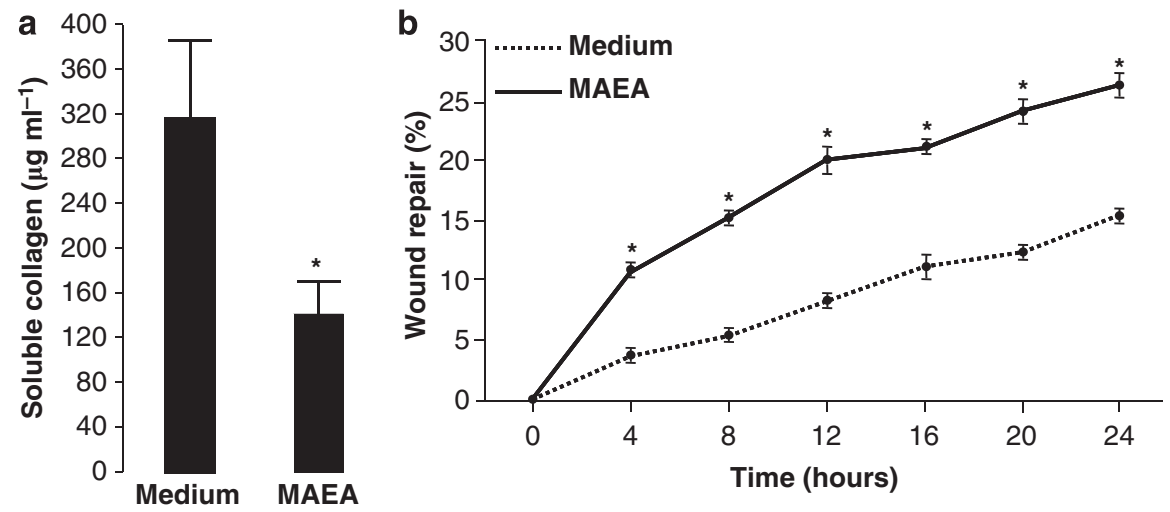

Figure 5 In vitro effects of methanandamide (MAEA) on myofibroblasts isolated from the submucosa of strictured areas of 11 patients with fibrostenosing Crohn's disease. Myofibroblasts were cultured with medium alone or with medium containing $0.1 \mu \mathrm{m}$ MAEA. (a) Soluble collagen concentration in myofibroblast culture supernatants. Results are mean \pm s.d. ${ }^{*} P<0.01$. (b) Myofibroblast migration, assessed by in vitro wound-healing scratch assay, at $0,4,8,12,16,20$, and $24 \mathrm{~h}$. Results, which are expressed as percentage of wound repair, are mean \pm s.d. ${ }^{\S} P<0.05 \mathrm{vs}$. medium at $4,8,12,16,20$, and $24 \mathrm{~h}$.

as shown by the dose-dependent inhibition of IFN- $\gamma$ and IL-17 production by $\mathrm{CD}^{+}{ }^{+}$LPMCs. These data support a clear role for the endocannabinoid system in downregulating inflammation in IBD. ${ }^{32}$ As the activated form of STAT4 (i.e., pSTAT4) and T-bet are essential mediators of naive T-cell polarization toward the Th1 phenotype, ${ }^{33}$ we explored the effect of MAEA on these two transcription factors in anti-CD3/CD28-stimulated LPMCs from inflamed areas of IBD patients. MAEA significantly inhibited the anti-CD3/CD28-induced upregulation of both T-bet and PSTAT4, further supporting its role in dampening the Th1 immune response. Moreover, the inhibitory effect of MAEA on TNF- $\alpha$ production by total LPMCs suggests that not only $\mathrm{T}$ cells, but also monocytes and macrophages, which are the major source of TNF- $\alpha,{ }^{2}$ are susceptible to cannabinoid action.

Of further interest is the fact that the endocannabinoid system plays a role in antagonizing the fibrogenic process in chronic liver disease. ${ }^{34}$ Indeed, CB2 stimulation triggers potent antifibrogenic in vitro effects, such as growth inhibition and apoptosis, on hepatic stellate cells and myofibroblasts from patients with active cirrhosis. ${ }^{34}$ Additionally, CB2 knockout mice are more sensitive than wild-type littermates to bleomycin-induced dermal fibrosis. ${ }^{35}$ As $>30 \%$ of patients with Crohn's disease develop intestinal fibrosis leading to stricture formation, frequently requiring surgical resection, ${ }^{36}$ and myofibroblasts are key cells in the process of wound healing and stricture formation in the gut, ${ }^{37}$ we investigated the in vitro effects of MAEA on myofibroblasts isolated from intestinal Crohn's disease strictures. We found that MAEA reduced collagen production by strictured Crohn's disease myofibroblasts and increased their migration ability, thus supporting the view that endocannabinoid pathways might be involved in the fibrogenic process in Crohn's disease.

\section{METHODS}

Patients and tissues. Endoscopic biopsies or surgical specimens were taken from macroscopically and microscopically inflamed intestinal mucosa or strictured areas of 41 patients affected by Crohn's disease
(Table 2a) and 33 patients affected by ulcerative colitis (Table $2 \mathbf{b}$ ). Diagnosis of Crohn's disease was ascertained according to the usual clinical criteria, ${ }^{38}$ and the site and extent of the disease were confirmed by endoscopy, histology, and enteroclysis in all patients. Diagnosis of ulcerative colitis was ascertained according to the usual clinical criteria, ${ }^{39}$ and the site and extent of the disease were confirmed by endoscopy and histology. None of the IBD patients had been ever treated with cyclosporine, methotrexate, infliximab, or adalimumab. Mucosal samples were also collected from the colon of 16 subjects who turned out to have functional diarrhea at the end of their diagnostic work-up (mean age 40.5 years, range 25-63), and from macroscopically and microscopically unaffected colonic areas of 18 patients undergoing colectomy for colon cancer (mean age 47.8 years, range $41-70$ ). Each patient who took part in the study gave informed consent, and ethics committee approval was obtained.

HPLC-tandem mass spectrometry. Biopsies were subjected to lipid extraction with chloroform/methanol $(2: 1, \mathrm{v} / \mathrm{v})$, in the presence of $\mathrm{d}_{8}-\mathrm{AEA}, \mathrm{d}_{8}-2-\mathrm{AG}$, and $\mathrm{d}_{4}$-PEA, as internal standards. ${ }^{40}$ The organic phase was dried, pre-purified by chromatography on silica, and then analyzed by HPLC electrospray ionization mass spectrometry using an LCQ Duo quadrupole ion trap mass spectrometry (ThermoFinnigan, San Jose, CA) with a TSP4000 HPLC pump and a TSP AS3000 autosampler. Quantitative analysis was performed by selected ion recording over the respective protonated molecular ions.

Radiochromatographic method. The synthesis of $\left[{ }^{3} \mathrm{H}\right]$ AEA through the activity of NAPE-PLD (E.C. 3.1.4.4) was assayed in tissue homogenates $\left(100 \mu \mathrm{g}\right.$ per test), using $100 \mu \mathrm{M}\left[{ }^{3} \mathrm{H}\right] \mathrm{NArPE},{ }^{41}$ by means of reversedphase HPLC, as reported. ${ }^{42}$ The hydrolysis of $\left[{ }^{3} \mathrm{H}\right]$ AEA by FAAH was assayed in tissue homogenates ( $50 \mu \mathrm{g}$ per test) by measuring the release of $\left[{ }^{3} \mathrm{H}\right]$ ethanolamine, as reported. ${ }^{43}$ AEA was from Sigma-Aldrich (St Louis, MO), whereas $\left[{ }^{3} \mathrm{H}\right]$ AEA $\left(60 \mathrm{Ci} \mathrm{mmol}^{-1}\right)$ and $N-\left[{ }^{3} \mathrm{H}\right]$ arachidonoyl-phosphatidylethanolamine $\left(\left[{ }^{3} \mathrm{H}\right] \mathrm{NArPE}, 200 \mathrm{Ci} \mathrm{mmol}^{-1}\right)$ were from ARC (St Louis, MO).

Cell isolation and culture. LPMCs were isolated as previously described. ${ }^{44}$ Briefly, the epithelial layer was removed with $1 \mathrm{~mm}$ EDTA (Sigma-Aldrich). After stirring for $1 \mathrm{~h}$ at $37^{\circ} \mathrm{C}$, the supernatant was removed and the remaining tissue was treated with type $1 \mathrm{~A}$ collagenase $\left(1 \mathrm{mg} \mathrm{ml}^{-1}\right.$; Sigma-Aldrich) for $2 \mathrm{~h}$ with stirring at $37^{\circ} \mathrm{C}$. The crude cell suspension was allowed to stand for $5 \mathrm{~min}$ to permit debris 
Table 2 Clinical features of patients with (a) CD ( $n=41)$ and (b) UC ( $n=33)$

\begin{tabular}{lc}
\hline Characteristics and parameters & N \\
\hline (a) & \\
Age (years) & 7 \\
First attack & \\
Intestinal location & \\
$\quad$ Small bowel and colon & 26 \\
Colon only & 15 \\
& \\
Disease behavior & \\
Fistulizing & \\
Stricturing & 7 \\
Luminal & 11 \\
& 23
\end{tabular}

$$
\begin{gathered}
57.3(1-212) \\
1.8(0-5) \\
203(69-405)
\end{gathered}
$$

\section{Treatment \\ Mesalazine \\ Mesalazine + topical steroids \\ Mesalazine + antibiotics \\ Mesalazine + azathioprine/ \\ 6-mercaptopurine}

Azathioprine/6-mercaptopurine

(b)

Age (years)

First attack

6

\section{Intestinal location \\ Pancolitis \\ Left-sided colitis \\ Duration of disease (months) \\ Number of recurrences \\ Clinical Activity Index}

\section{Treatment}

\section{Mesalazine}

Mesalazine + topical steroids

11

Mesalazine + azathioprine/

6-mercaptopurine

Abbreviations: CD, Crohn's disease; CDAl, Crohn's Disease Activity Index; UC, ulcerative colitis.

sedimentation. Cells from the supernatant were washed twice, resuspended in $1 \mathrm{ml}$ RPMI-1640 medium (Sigma-Aldrich) containing 10\% fetal bovine serum, $100 \mathrm{U} \mathrm{ml}^{-1}$ penicillin, and $100 \mu \mathrm{g} \mathrm{ml}^{-1}$ streptomycin, and kept on ice until used. Purified $\mathrm{CD}^{+}$cells were obtained by positive magnetic-activated cell sorting separation using a $\mathrm{CD}^{+}$cell isolation kit (Miltenyi Biotec, Auburn, CA) after removing monocytes by adherence. The resulting cell preparation contained $>95 \% \mathrm{CD}^{+} \mathrm{T}$ cells as assessed by flow cytometry. Cells were not used if viability was $<90 \%$. Isolated LPMCs or $\mathrm{CD}^{+} \mathrm{T}$ cells $\left(2 \times 10^{5}\right.$ cells per well $)$ were stimulated in anti-CD3-coated 96-well plates (BD Biosciences, Oxford, UK) with antiCD28 antibody $\left(0.5 \mu \mathrm{g} \mathrm{ml}^{-1}\right.$, eBioscience, San Diego, CA), and incubated for $48 \mathrm{~h}$ with medium containing MAEA $(0.1,1,10 \mu \mathrm{M}$; Calbiochem, San Diego, CA) or FK506 (10 $\mu \mathrm{M}$; Sigma-Aldrich). As negative control, $0.1 \%$ dimethylsulfoxide (Sigma-Aldrich) was used. After culture, supernatants were snap frozen and stored at $-70^{\circ} \mathrm{C}$. Myofibroblasts were isolated from uninflamed mucosa overlying Crohn's disease strictures as previously described. ${ }^{45}$ Briefly, after removing the epithelial layer with EDTA treatment, tissue samples were denuded of epithelial cells, and were subsequently cultured at $37^{\circ} \mathrm{C}$ in a humidified $\mathrm{CO}_{2}$ incubator in Dulbecco's modied Eagle's medium (Sigma-Aldrich) supplemented with 20\% fetal bovine serum, $1 \%$ nonessential amino acids (Invitrogen, Paisley, UK), $100 \mathrm{U} \mathrm{ml}^{-1}$ penicillin, $100 \mu \mathrm{g} \mathrm{ml}^{-1}$ streptomycin, $50 \mu \mathrm{g} \mathrm{ml}^{-1}$ gentamycin, and $1 \mu \mathrm{g} \mathrm{ml}^{-1}$ amphotericin (Sigma-Aldrich). During culture, numerous cells appeared both in suspension and adherent to the culture dish. The cells in suspension were removed every $24-$ to $72-\mathrm{h}$ culture period, and the denuded tissue was maintained in culture for up to 6 weeks. Established colonies of myofibroblasts were seeded into $25 \mathrm{~cm}^{2}$ culture flasks and cultured in Dulbecco's modied Eagle's medium supplemented with $20 \%$ fetal bovine serum and antibiotics. At confluence, the cells were passaged using trypsin-EDTA in a 1:2 to 1:3 split ratio. Cells were grown up to at least passage 4 before there were enough to be used in stimulation experiments with MAEA, and were characterized by immunocytochemical staining as previously described. ${ }^{45}$

Wound-healing scratch assay. Myofibroblast migration was assessed according to the method of Rodriguez et al. ${ }^{46}$ as modified by us. ${ }^{47}$ Briefly, cells $\left(2 \times 10^{5}\right)$ were seeded into Nunc cell culture dishes (Nalge Nunc International, Rochester, NY) with $2 \mathrm{~mm}$ grids, size $35 \times 10 \mathrm{~mm}$, in $2 \mathrm{ml}$ of Dulbecco's modied Eagle's medium supplemented with $20 \%$ fetal bovine serum and antibiotics. The cells were maintained at $37^{\circ} \mathrm{C}$ and $5 \%$ $\mathrm{CO}_{2}$ until confluent. Once confluent, each dish of monolayer cells was given a mechanical wound by scoring with a $200 \mu$ l pipette tip, parallel to the grid bars along the central grid line. This permits easy viewing of the cells growing back together, and ensures that the $2 \mathrm{~mm}$ grid may be used as a reference, so that the wound areas can be measured and compared. Wound placement was checked with an Olympus inverted CK2 microscope (Olympus UK, London, UK). The medium was then removed, and the cells were washed five times with HL-1 serum-free medium (Cambrex Bio Science, Nottingham, UK) supplemented with antibiotics, and then replaced with $1.5 \mathrm{ml} \mathrm{HL}-1$ medium with $0.1 \%$ dimethylsulfoxide (Sigma-Aldrich) or MAEA $0.1 \mu$ M. Photographs of the cells in each grid along the induced wound were taken at $0,4,8,12,16,20$, and $24 \mathrm{~h}$, using a digital camera (Olympus Camedia $34-40$ zoom, $\times 20$ magnification) attached to a light microscope. The computer program "Image J" was used to measure the area of initial damage (images taken at time 0 ) and of the remaining damage at subsequent time points. Each grid image was observed separately, and two points per grid at the same position at every time point were measured using imaging software at the same magnification. The percentage of wound repair was then calculated.

Organ culture. Biopsy specimens were placed on iron grids in the central well of an organ culture dish and the dishes placed in a tight chamber with $95 \% \mathrm{O}_{2} / 5 \% \mathrm{CO}_{2}$ at $37^{\circ} \mathrm{C} .{ }^{45}$ Increasing concentrations of MAEA ( 1 and $10 \mu \mathrm{M}$ ) were added to the medium containing RPMI-1640 medium supplemented with $10 \% \mathrm{HL}-1$ (Cambrex Bio Science), $100 \mathrm{U} \mathrm{ml}^{-1}$ penicillin, and $100 \mu \mathrm{g} \mathrm{ml}^{-1}$ streptomycin. As negative control, $0.1 \%$ dimethylsulfoxide (Sigma-Aldrich) was used. After $24 \mathrm{~h}$ culture, supernatants were snap frozen and stored at $-70^{\circ} \mathrm{C}$.

Western blotting. Western blotting was performed according to a modified method described previously. ${ }^{47}$ In brief, tissue samples or cells were lysed in ice-cold lysis buffer (10 mm EDTA, $50 \mathrm{~mm}$ pH 7.4 Tris- $\mathrm{HCl}$, $150 \mathrm{~mm}$ sodium chloride, $1 \%$ Triton-X-100, $2 \mathrm{~mm}$ phenylmethylsulfonyl 
fluoride, $2 \mathrm{~mm}$ sodium orthovanadate, $10 \mathrm{mg} \mathrm{ml}^{-1}$ leupeptin, and $2 \mathrm{mg} \mathrm{ml}^{-1}$ aprotinin) and the amount of protein was determined by the Bio-Rad Protein assay (Bio-Rad Laboratories, Hemel Hempstead, $\mathrm{UK})$. An equal amount of protein $(20 \mu \mathrm{g})$ was loaded onto $10 \%$ sodium dodecyl sulfate-polyacrylamide gels and blotted onto polyvinylidene fluoride sheets (Amersham Biosciences, Piscataway, NJ) under reducing conditions. Membranes were blocked with $10 \%$ nonfat dried milk and $5 \%$ bovine serum albumin for $2 \mathrm{~h}$, and then incubated overnight with rabbit anti-CB1 (diluted 1:500, Cayman Chemicals, Ann Arbor, MI), rabbit anti-CB2 (1:300, Cayman Chemicals), and mouse anti- $\beta$-actin $(1: 1,000)$, mouse anti-STAT4 $(1: 500)$, mouse anti-phospho-STAT4 (pSTAT4, 1:500), and mouse anti-T-bet (1:500), all from Santa Cruz Biotechnology (Santa Cruz, CA). Membranes were rinsed and incubated with the appropriate horseradish peroxidase-conjugated secondary antibody $(1: 2,000)$ in blocking solution. Detection was performed using West Dura Chemiluminescence System (Pierce, Rockford, IL).

Immunohistochemistry. Tissue blocks were fixed in $4 \%$ buffered formaldehyde and embedded in paraffin. Blocks were cut into longitudinal 4-mm thick sections that were mounted on glass slides with the positively charged surface and air-dried. After blocking endogenous peroxidase with $0.3 \%$ hydrogen peroxide for $8 \mathrm{~min}$, proteolytic digestion was obtained with trypsin $2 \%$ in calcium chloride $0.1 \%, \mathrm{pH} 7.8$, for $5 \mathrm{~min}$. Sections were then immersed in $0.01 \mathrm{M}$ citrate buffer $(\mathrm{pH}=6.0)$ and microwavetreated for $10 \mathrm{~min}$ at $410 \mathrm{~W}$. After washing in phosphate-buffered saline, sections were incubated with an anti-CB1 antibody (Abcam, Cambridge, UK; ab23703; 1:100 dilution) or an anti-CB2 antibody (Abcam; ab45942; 1:100 dilution) overnight at $4^{\circ} \mathrm{C}$, and then treated with a secondary biotinylated antibody and peroxidase-conjugated streptavidin (Biogenex, San Ramon, CA). Sections were then dehydrated in ethanol, cleared in xylene, and coverslipped with Eukitt mounting medium (Kindler $\mathrm{GmBH}$, Freiburg, Germany).

ELISA. IFN- $\gamma$, TNF- $\alpha$, and IL-17 were measured in culture supernatants using human IFN- $\gamma$ ELISA, (Endogen, Woburn, MA), and human TNF- $\alpha$ Quantikine ELISA Kit and human IL-17 Quantikine ELISA Kit, both from R\&D Systems (Abingdon, UK), according to the manufacturer's instructions. In addition, anti-CB1 (diluted 1:500) and anti-CB2 (diluted 1:300) antibodies were used to determine CB1 and CB2 protein expression by ELISA in mucosal homogenates. The $\mathrm{A}_{405}$ values in unknown samples $(20 \mu \mathrm{g}$ protein per well) were within the linearity range of a calibration curve drawn with different amounts of homogenates (in the range $0-40 \mu \mathrm{g}$ protein per well). The results were expressed as mean percentages $( \pm$ s.d.) of control subjects.

Collagen assay. Total soluble collagen was measured using Sircol Collagen Assay kit (Biocolor, Belfast, UK) according to the manufacturer's instructions. The Sircol dye reagent has been formulated to bind specifically to the [Gly-X-Y]n helical structure found in collagen types I to XIV. The collagen content in each sample of cell supernatant was obtained as an average of three readings.

Statistical analysis. Data were analyzed in the GraphPad Prism statistical PC program (GraphPad Software, San Diego, CA) using the paired $t$-test and the Mann-Whitney $U$-test. A level of $P<0.05$ was considered statistically significant.

\section{ACKNOWLEDGMENTS}

This project was supported in part by the European Union Framework Programme 7 collaborative project grant IPODD (call: FP7-Health-2007-A) and by Fondazione TERCAS (2009-2012 project) to M.M.

\section{DISCLOSURE}

The authors declared no conflict of interest.

\section{REFERENCES}

1. Bouma, G. \& Strober, W. The immunological and genetic basis of inflammatory bowel disease. Nat. Rev. Immunol. 3, 521-533 (2003).

2. MacDonald, T.T. \& Monteleone, G. Immunity, inflammation, and allergy in the gut. Science 307, 1920-1925 (2005).

3. Gordon, J.N., Di Sabatino, A. \& MacDonald, T.T. The pathophysiologic rationale for biological therapies in inflammatory bowel disease. Curr. Opin. Gastroenterol. 21, 431-437 (2005).

4. Kunos, G. \& Pacher, P. Cannabinoids cool the intestine. Nat. Med. 10, 678-679 (2004).

5. Izzo, A.A. \& Camilleri, M. Emerging role of cannabinoids in gastrointestinal and liver diseases: basic and clinical aspects. Gut 57, 1140-1155 (2008).

6. Storr, M.A. et al. Targeting endocannabinoid degradation protects against experimental colitis in mice: involvement of CB1 and $\mathrm{CB} 2$ receptors. J. Mol. Med. 86, 925-936 (2008).

7. Muccioli, G.G. Endocannabinoid biosynthesis and inactivation, from simple to complex. Drug. Discov. Today 15, 474-483 (2010).

8. Petrosino, S., Ligresti, A. \& Di Marzo, V. Endocannabinoid chemical biology: a tool for the development of novel therapies. Curr. Opin. Chem. Biol. 13, 309-320 (2009).

9. Pertwee, R.G. et al. Cannabinoid receptors and their ligands: beyond CB1 and CB2. Pharmacol. Rev. 62, 588-631 (2010).

10. De Petrocellis, L. \& Di Marzo, V. Non-CB1, non-CB2 receptors for endocannabinoids, plant cannabinoids, and synthetic cannabimimetics: focus on G-protein-coupled receptors and transient receptor potential channels. J. Neuroimmune. Pharmacol. 5, 103-121 (2010).

11. Solorzano, C. et al. Selective N-acylethanolamine-hydrolyzing acid amidase inhibition reveals a key role for endogenous palmitoylethanolamide in inflammation. Proc. Natl. Acad. Sci. USA 106, 20966-20971 (2009).

12. Ueda, N., Tsuboi, K. \& Uyama, T. Enzymological studies on the biosynthesis of $\mathrm{N}$-acylethanolamines. Biochim. Biophys. Acta. 1801, 1274-1285 (2010).

13. Petrosino, S. \& Di Marzo, V. FAAH and MAGL inhibitors: therapeutic opportunities from regulating endocannabinoid levels. Curr. Opin. Investig. Drugs 11, 51-62 (2010).

14. Fezza, F., De Simone, C., Amadio, D. \& Maccarrone, M. Fatty acid amide hydrolase: a gate-keeper of the endocannabinoid system. Subcell. Biochem. 49, 101-132 (2008).

15. Izzo, A.A. \& Sharkey, K.A. Cannabinoids and the gut: new developments and emerging concepts. Pharmacol. Ther. 126, 21-38 (2010).

16. Tanasescu, R. \& Constantinescu, C.S. Cannabinoids and the immune system: an overview. Immunobiology 215, 588-597 (2010).

17. Cencioni, M.T. et al. Anandamide suppresses proliferation and cytokine release from primary human T-lymphocytes mainly via CB2 receptors. PLoS One 5, e8688 (2010).

18. Maccarrone, M. et al. Lipopolysaccharide downregulates fatty acid amide hydrolase expression and increases anandamide levels in human peripheral lymphocytes. Arch. Biochem. Biophys. 393, 321-328 (2001).

19. Smid, S.D. Gastrointestinal endocannabinoid system: multifaceted roles in the healthy and inflamed intestine. Clin. Exp. Pharmacol. Physiol. 35, 1383-1387 (2008).

20. Massa, F. et al. The endogenous cannabinoid system protects against colonic inflammation. J. Clin. Invest. 113, 1202-1209 (2004).

21. Marquéz, L. et al. Ulcerative colitis induces changes on the expression of the endocannabinoid system in the human colonic tissue. PLoS One 4, e6893 (2009).

22. D'Argenio, G. et al. Up-regulation of anandamide levels as an endogenous mechanism and a pharmacological strategy to limit colon inflammation. FASEB J. 20, 568-570 (2006).

23. Di Marzo, V. \& Izzo, A.A. Endocannabinoid overactivity and intestinal inflammation. Gut 55, 1373-1376 (2006).

24. Klein, T.W. Cannabinoid-based drugs as anti-inflammatory therapeutics. Nat. Rev. Immunol. 5, 400-411 (2005).

25. Lunn, C.A., Reich, E.P. \& Bober, L. Targeting the CB2 receptor for immune modulation. Expert. Opin. Ther. Targets 10, 653-663 (2006).

26. Rieder, S.A., Chauhan, A., Singh, U., Nagarkatti, M. \& Nagarkatti, P. Cannabinoid-induced apoptosis in immune cells as a pathway to immunosuppression. Immunobiology 215, 598-605 (2010).

27. Börner, C., Höllt, V. \& Kraus, J. Activation of human T cells induces upregulation of cannabinoid receptor type 1 transcription. Neuroimmunomodulation 14, 281-286 (2007). 
28. Wright, K.L., Duncan, M. \& Sharkey, K.A. Cannabinoid CB2 receptors in the gastrointestinal tract: a regulatory system in states of inflammation. $\mathrm{Br}$. J. Pharmacol. 153, 263-270 (2008).

29. Croxford, J.L. \& Yamamura, T. Cannabinoids and the immune system: potential for the treatment of inflammatory diseases? J. Neuroimmunol. 166, 3-18 (2005).

30. Wacnik, P.W. et al. Cannabinoids affect dendritic cell (DC) potassium channel function and modulate DC T cell stimulatory capacity. J. Immunol. 18, 3057-3066 (2008).

31. Rovedatti, L., Kudo, T. \& Biancheri, P. et al. Differential regulation of interleukin-17 and interferon- $\gamma$ production in inflammatory bowel disease. Gut 58, 1629-1636 (2009).

32. Engel, M.A. et al. Ulcerative colitis in AKR mice is attenuated by intraperitoneally administered anandamide. J. Physiol. Pharmacol. 59, 673-689 (2008).

33. Thieu, V.T. et al. Signal transducer and activator of transcription 4 is required for the transcription factor T-bet to promote Thelper 1 cell-fate determination. Immunity 29, 679-690 (2008).

34. Julien, B. et al. Antifibrogenic role of the cannabinoid receptor CB2 in the liver. Gastroenterology 128, 742-755 (2005).

35. Akhmetshina, A. et al. The cannabinoid receptor CB2 exerts antifibrotic effects in experimental dermal fibrosis. Arthritis Rheum 60,1129-1136 (2009).

36. Burke, J.P. et al. Fibrogenesis in Crohn's disease. Am. J. Gastroenterol. 102, 438-448 (2007).

37. Powell, D.W. et al. Myofibroblasts. II. Intestinal subepithelial myofibroblasts. Am. J. Physiol. 277, C183-C201 (1999).

38. Best, W.R. et al. Development of a Crohn's disease activity index: National Cooperative Crohn's Disease Study. Gastroenterology 70, 439-444 (1976).
39. Rachmilewitz, D. Coated mesalazine (5-aminosalicylic acid) versus sulphasalazine in the treatment of active ulcerative colitis: a randomized study. BMJ 298, 82-86 (1989).

40. Francavilla, F. et al. Characterization of the endocannabinoid system in human spermatozoa and involvement of transient receptor potential vanilloid 1 receptor in their fertilizing ability. Endocrinology 150, 4692-4700 (2009).

41. Okamoto, Y. et al. Molecular characterization of a phospholipase D generating anandamide and its congeners. J. Biol. Chem. 279, 5298-5305 (2004).

42. Fezza, F. et al. Radiochromatographic assay of N-acyl-phosphatidylethanolamine-specific phospholipase D activity. Anal. Biochem. 339, 113-120 (2005).

43. Fegley, D. et al. Characterization of the fatty acid amide hydrolase inhibitor cyclohexyl carbamic acid 3' -carbamoyl-biphenyl-3-yl ester (URB597): effects on anandamide and oleoylethanolamide deactivation. J. Pharmacol. Exp. Ther. 313, 352-358 (2005).

44. Di Sabatino, A. et al. Targeting gut $\mathrm{T}$ cell $\mathrm{Ca}^{2+}$ release-activated $\mathrm{Ca}^{2+}$ channels inhibits Th1 cytokine production and T-box transcription factor T-bet in inflammatory bowel disease. J. Immunol. 183, 3454-3462 (2009).

45. Di Sabatino, A. et al. Functional modulation of Crohn's disease myofibroblasts by anti-TNF antibodies. Gastroenterology 133, 137-149 (2007).

46. Rodriguez, L.G., Wu, X. \& Guan, J.L. Wound-healing assay. Methods Mol. Biol. 294, 23-99 (2005).

47. Di Sabatino, A. et al. Transforming growth factor- $\beta$ signalling and matrix metalloproteinases in the mucosa overlying Crohn's disease strictures. Gut 58, 777-789 (2009). 